\title{
Do we have an acceptable model of power-law creep?
}

\author{
F.R.N. Nabarro ${ }^{a, b, *}$ \\ ${ }^{a}$ Condensed Matter Physics Research Group, School of Physics, University of the Witwatersrand, Private Bag 3, Johannesburg WITS 2050, South Africa \\ ${ }^{\mathrm{b}}$ Division of Manufacturing and Materials, CSIR, Box 395, Pretoria 0001, South Africa
}

Received 25 August 2003; received in revised form 18 September 2003; accepted 22 September 2003

\begin{abstract}
Three models of power-law creep are frequently presented. Those of Weertman assume distributed sources of dislocations which spread until they meet dislocations from other sources. They then annihilate by bulk diffusion. If the density of sources is independent of stress, a $9 / 2$ power law follows, but the creep rate is grossly less than that observed. Moreover, the more plausible assumption of a source density proportional to the cube of the stress leads to the conventional power law of 3 . The model of Spingarn and Nix assumes dislocation glide with pile-ups at the grain boundaries. These cause steps on the boundaries, which are removed by grain-boundary diffusion. A fifth power law follows. The agreement in absolute creep rate shown in the original paper arises from a misreading of the tabulated data, and the true predicted creep rate is again far too low. Vacancy diffusion along dislocation cores in the interior of the grains is not likely to dominate grain-boundary diffusion, because the cross section of the dislocation network exceeds that of the grain boundary material only at large grain sizes. While Mecking and Estrin have shown that the vacancy concentration produced mechanically even in dislocation cell walls is likely to exceed that in thermal equilibrium only below $1 / 2 T_{\mathrm{m}}$, it seems possible that the highly mobile interstitials produced mechanically may migrate along dislocations of secondary glide systems to allow vacancy and interstitial dipoles of the primary system to annihilate mutually, with a probable power law of 5. However, the observations of Andrade and of Hanson, which may apply to the regime of power-law breakdown rather than power-law creep, strongly indicate that deformation is localized at the grain boundaries. If this is the case, existing theories of power-law creep based on models of homogeneous deformation are irrelevant; alternatively, the regimes of power-law creep and power-law breakdown must be treated separately.
\end{abstract}

(C) 2004 Published by Elsevier B.V.

Keywords: Creep mechanisms; Power-law creep; Creep power-law breakdown

\section{Introduction}

The shape of the curve describing the elongation of a pure polycrystalline metal under a tensile stress as a function of time depends on the stress and on the temperature. Over a wide range of these parameters, the curve can be resolved fairly clearly into four stages.

Stage 1 is that of an initial rapid extension.

Stage 2 is well described by Andrade's law: the strain $\varepsilon$ depends on the time $t$ according to the relation:

$\varepsilon=\beta t^{1 / 3}$

Stage 3 is that of the so-called steady-state creep:

$$
\varepsilon=\kappa t
$$

* Tel.: +27 11717 6875; fax: +27 117176879 .

E-mail address: nabarrof@physics.wits.ac.za (F.R.N. Nabarro).
The final stage 4 is one of rapidly accelerating creep. The process is complicated, and occurs when the material has ceased to serve a useful engineering purpose. We shall not consider it further.

Attention is usually focused on steady-state creep in stage 3. A theoretical understanding of this stage is particularly important because most of the strain in a laboratory test of normal duration usually occurs in the earlier stages, or else at temperatures or stresses higher than those present in service.

At a given temperature, the rate of steady-state creep $\dot{\varepsilon}$ is a rapidly increasing function of stress $\sigma$. Very often, a power-law dependence:

$\dot{\varepsilon}=C \sigma^{n}$

is observed, with $n$ between 4 and 5. This is called power-law creep. At rather higher stresses the creep rate increases more rapidly with stress. This is called the regime of power-law breakdown. The creep rate is often an exponential function 
of the stress. There is little evidence whether the physical processes occurring in the power-law region and the region of power-law breakdown are the same or different. Since detailed metallographic studies are usually carried out over a short time period, it turns out that some of them relate to the borderline between these two regions, or to the region of power-law breakdown. With the exception of the model of Spingarn and Nix [1], theories of power-law creep have been based on the tacit assumption that creep in polycrystals represents essentially a homogeneous deformation of each grain, so that problems of the conformity of adjacent grains do not arise. In the model of Spingarn and Nix, the rate-controlling process occurs in the grain boundaries, but the strain occurs predominantly by homogeneous deformation of the grains. The discussion in Section 2 suggests that none of these models can satisfactorily predict rates of strain as large as these observed. We therefore turn in Section 3 to the metallographic evidence. While this bears more on the region of power-law breakdown than on that of power-law creep, it strongly suggests a model in which the grain boundaries are undulating with an amplitude of the order of a micron.

\section{The existing theoretical models}

In this section we examine theoretical models which are often quoted. The conclusion is that every model either lacks physical plausibility or fails to predict a rate of creep of the order which is observed experimentally. The theoretical and experimental data are compared for the case of polycrystalline cold-drawn copper wire creeping at a temperature $T=688 \mathrm{~K}$ under a tensile stress of $1.5 \mathrm{MPa}$.

The theoretical estimates require a knowledge of the bulk diffusion coefficient $D_{\mathrm{b}}$ at that temperature, and of the product of the grain-boundary diffusion coefficient $D_{\mathrm{gb}}$ and the effective thickness $\delta$ of the grain boundary.

At $T=688 \mathrm{~K}$, we take, from the data of [2], $D_{\mathrm{b}}=$ $1.138 \times 10^{-20} \mathrm{~m}^{2} \mathrm{~s}^{-1}$.

Surholt and Herzig [3] showed that impurities reduced the rate of grain-boundary self-diffusion in copper. Since creep experiments are not usually performed on material of the highest purity, we take the appropriate value of the grain-boundary diffusivity to be in the region of the two low-lying results shown in their Fig. 4 . At $T=688 \mathrm{~K}$, this implies:

$D_{\mathrm{gb}} \delta=1.55 \times 10^{-22} \mathrm{~m}^{3} \mathrm{~s}^{-1}$

\subsection{The model of Weertman [4,5]}

In this model, creep deformation occurs by the glide of dislocations from sources with a density $M\left(\mathrm{~m}^{-3}\right)$. Dislocations spread from these sources until they are blocked by dislocations spreading from other sources, forming edge dislocation dipoles. These dipoles are removed by bulk diffu- sion. The resulting creep rate is of the form:

$\dot{\varepsilon}=\alpha^{*} D_{\mathrm{b}}\left(\frac{\sigma}{\mu}\right)^{3.5} \frac{\sigma \Omega}{k T}$

where $\sigma$ is the applied tensile stress, $\mu$ the shear modulus 4.4 $\times 10^{10} \mathrm{~Pa}, \Omega$ the atomic volume and $k$ Boltzmann's constant. The factor $\alpha^{*}$ is a numerical multiple of $b^{-3.5} M^{-1 / 2}$.

If $M$ is assumed to be independent of $\sigma$, the predicted dependence on stress is as $\sigma^{4.5}$, in close agreement with observation. However, studies of plastic deformation controlled by dislocation glide usually conform rather well to the principle of similitude, according to which the dislocation pattern remains self-similar during plastic deformation, with a linear scale proportional to $1 / \sigma$. If similitude is obeyed, $M$ $\propto \sigma^{3}$ and the strain rate of Eq. (5) obeys the "natural law":

$\dot{\varepsilon} \propto \sigma^{3}$

Weertman (1968) [5] states that numerical agreement with observations of power-law creep with an exponent 4.5 requires $M$ to be in the range $3.6 \times 10^{14}$ to $1.8 \times 10^{17} \mathrm{~m}^{-3}$, which is reasonable at a stress of $\sigma=10^{5} \mu=0.44 \mathrm{MPa}$. The creep tests of Brown et al. [6] were made on cold drawn copper in the stress range 18-55 MPa. If one takes the dislocation cell size to be that corresponding to the formation stress of $30 \mathrm{MPa}$, and to be given roughly by the formula $10 \mu b / \sigma$, where $b$ is the Burgers vector, $2.556 \times 10^{-10} \mathrm{~m}$ and $\mu$ the shear modulus, this cell size is $3.7 \times 10^{-6} \mathrm{~m}$, while the separation between sources corresponding to the proposed value of $M$ is $1.4 \times 10^{-5}$ to $1.8 \times 10^{-6} \mathrm{~m}$. This suggests that there would usually be about one effective source per cell, implying that the model can give the observed creep rate only by assuming an implausibly low value of $M$.

\subsection{The model of Weertman [7]}

This model involves consideration of two dislocation configurations, Fig. 2 and 3 in Weertman's paper, reproduced here as Figs. 1 and 2, with the addition of sets of coordinate axes and labeling of some dislocation nodes.

In Fig. 1, the dislocation segment EF lies in the plane AEFD with Burgers vector along $\pm y$. It cannot be a screw segment, as it is labeled.

In Fig. 2, the narrow edge dipoles again lie in $x-z$ planes with Burgers vectors along $\pm y$. The segments such as $\mathrm{AB}$ are correctly labeled as "jogged screws". It is not clear how the Burgers vectors are conserved at points such as A, where the three segments $\mathrm{AB}, \mathrm{AC}$ and $\mathrm{AD}$ meet. The model also has difficulty in predicting creep rates as high as those observed.

\subsection{The model of Spingarn and Nix}

In the model of Springarn and Nix, plastic deformation occurs by dislocation glide, on a single slip system in each grain. Cohesion between the grains is maintained by sliding on the grain boundaries, which is assumed not to be rate 


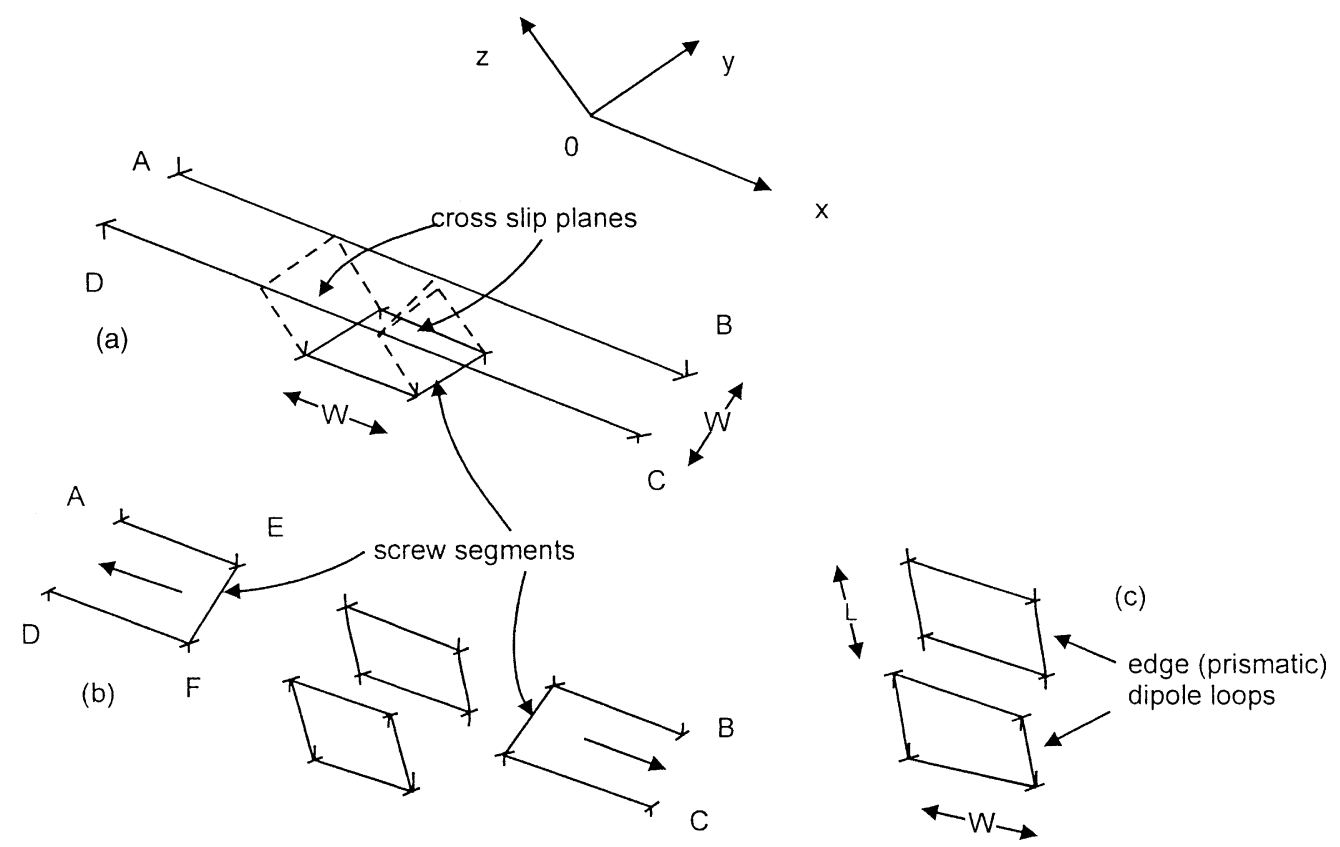

Fig. 1. Redrawn from [7]. The dislocation segments labeled as screws are in fact edges.

limiting if the grain boundaries are smooth. However, slip in each of two adjacent grains produces steps in their interface, and the rate-controlling process is the smoothing of these steps by diffusion along the grain boundary. The diffusion coefficient for grain-boundary diffusion is some $10^{8}$ times greater than that for diffusion in the bulk in the relevant temperature range of about half the melting temperature. The analysis leads simply and directly to a power-law exponent of 5. However, it predicts a creep rate proportional to the grain size, whereas the evidence [8] suggests that the creep rate decreases with increasing grain size up to about $100 \mu \mathrm{m}$, increasing only for grain sizes greater than $100 \mu \mathrm{m}$.

Moreover, the paper of Spingarn and Nix shows quite good numerical agreement between theory and observation for several systems. It turns out that this agreement arose from a misreading of the tabulated activation energies for

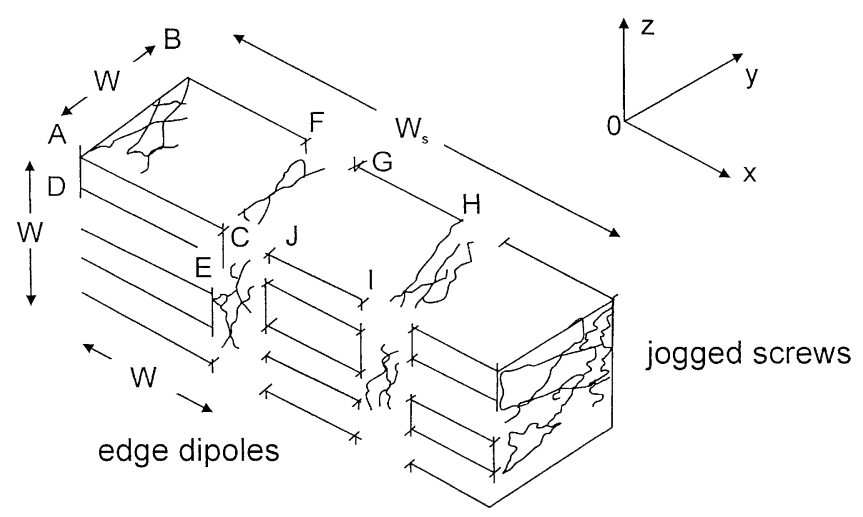

Fig. 2. Redrawn from [7]. Burgers vectors are not conserved at points such as A, B.... grain-boundary diffusion, which were listed in $\mathrm{kcal} \mathrm{mol}^{-1}$, but read as $\mathrm{kJ} \mathrm{mol}^{-1}$.

\subsection{A possible new model}

In essence, the rate-controlling process for creep in the matrix of a grain is the elimination of edge-dislocation dipoles. The dipoles of the primary slip system are likely to be equally frequently of vacancy and of interstitial character. Vacancy and interstitial dipoles will be linked by dislocations of secondary slip systems. These links allow vacancy and interstitial dipoles to annihilate by dislocation pipe diffusion. Interstitials produced by the creep deformation would be attracted to these dislocations, and very mobile along them. At high dislocation densities the rate of annihilation will be larger than that produced by other processes, possibly explaining the high observed creep rates and the low activation energy.

\section{What guidance does experiment give to a theoretical model?}

The theories discussed in Section 2 have attempted to explain the experimental observation that the creep rate is, over a wide range of stresses, closely proportional to a power of the stress, with an exponent of about 4.5-5.0. For polycrystalline copper the experiments of Brown et al. [6] show reasonable agreement with a power law of 5 in the temperature range $608-774 \mathrm{~K}$ and a stress range $13-56 \mathrm{MPa},\left(T / T_{\mathrm{m}}\right.$ $=0.45-0.57)\left(\sigma / \mu=2.4 \times 10^{-4}\right.$ to $\left.1.2 \times 10^{-3}\right)$, though their double-logarithmic plots are markedly curved. 


\subsection{The activation free energy}

It is sometimes regarded as almost axiomatic that the activation free energy for power-law creep is almost precisely that for self-diffusion in a pure metal. It is, moreover, claimed that the activation volume for creep (in the strict sense of $-k T$ times the rate of change of the natural logarithm of the creep rate with the hydrostatic pressure) is the same for both processes. Poirier [16] provided evidence that the two free energies agree in the case of six metals only at temperatures above about half the melting temperature, whereas power-law creep is usually observed below these temperatures. Sherby and Weertman [17] showed a graph in which the two free energies coincide extremely closely for 21 pure metallic phases, provided that the creep data are observed at a "sufficiently high" temperature for each metal. Their theoretical estimates of a "sufficiently high" temperature ranged from 0.28 to 1.27 times the melting temperature. Their summary of the experimental evidence seems rather convincing. This is discouraging theoretically, because the "natural law" for creep shows that the creep rate should be proportional to the coefficient of self-diffusion and to the cube of the stress. If diffusion occurs along dislocations, which have a density proportional to the square of the stress, the "natural law" becomes a creep rate proportional to the fifth power of the stress. The activation free energy then becomes that for self-diffusion along dislocations, which [18] is usually about $2 / 3$ of that for bulk diffusion in fcc metals. This is contrary to the evidence of Sherby and Weertman.

However, further analysis, supported by the data assembled by Luthy et al. [19], indicates clearly that, in the range $T / T_{\mathrm{m}} \leq 0.6$ where power-law creep is usually studied in pure metals, the activation energy for creep is usually substantially less than that for self-diffusion in the bulk.

\subsection{The activation volume}

Many observations show two rather well defined regions of the dependence of the steady-state creep rate on stress at moderate and high stresses. At moderate stresses, a relation of the form:

$\dot{\varepsilon}=A \sigma^{n} \exp \left(\frac{-Q}{R T}\right)$

is followed, with $n$ in the range 4.5-5, and $Q$ regarded as independent of stress. This is the power-law region. At higher stresses, the law is of the form:

$\dot{\varepsilon}=B \exp \left[\frac{\left(V^{\dagger} \sigma-U\right)}{k T}\right]$

where $B$, the activation volume $V^{\dagger}$ and $U$ are assumed independent of temperature and of stress. This is the form predicted by simple thermal-activation theory. Other observations fail to follow this pattern.
In copper, Andrade, Fig. 14 of [12], found on exact exponential dependence of creep rate on stress in the range 5-11 MPa at $683 \mathrm{~K}$, corresponding to an activation volume of $3.4 \times 10^{-27} \mathrm{~m}^{3}$, or 290 atomic volumes. Feltham and Meakin [9] found two regimes both exponential in the stress. The transition between the regimes depends on temperature, and seems to occur at a strain rate of about $10^{-5} \mathrm{~s}^{-1}$. At a temperature of $673 \mathrm{~K}$, in the low-stress range, their observations correspond to an activation volume of $9.7 \times 10^{-28} \mathrm{~m}^{3}$ or 82 atomic volumes, smaller than that of Andrade's observations, but still large. The re-plotting by Siethoff and Ahlborn [10] of the observations of Myshlyaev et al. [11] similarly shows two (or possibly three) regions of exponential dependence.

In the case of aluminium the many observations collected by Wu and Sherby [13] show a fairly sharp transition from a power-law to an exponential dependence. On the other hand, the data assembled by Vogler and Blum [14] show a systematic upward curvature in the log-log plot at each temperature, with a slope fairly close to 4 at $700 \mathrm{~K}\left(T / T_{\mathrm{m}}\right.$ $=0.75)$, but much greater than 5 at $400 \mathrm{~K}\left(T / T_{\mathrm{m}}=0.43\right)$.

Similarly, Moore et al. [15] found a linear relation between stress and logarithm of creep rate for a given temperature in lead.

The evidence for power-law creep in moderately pure fcc metals does not seem to be strong.

\subsection{Dependence of creep rate on grain size}

The observations of the dependence of creep rate on grain size in copper have been reviewed and extended by Wilshire and Palmer [8]. In the temperature range $723-753 \mathrm{~K}\left(T / T_{\mathrm{m}}\right.$ $=0.533-0.555)$, there is no clear dependence on grain size for grain sizes between 30 and $450 \mu \mathrm{m}$ at stresses above $40 \mathrm{MPa}$, where the stress exponent is 5 .

\subsection{The evidence from metallography}

There have been studies of creep in aluminium by Hanson and Wheeler [20] and by the school of Andrade, summarized in Andrade [12], of creep in several systems, mostly low-melting.

Most of the observations of Hanson and Wheeler were made under a tensile stress of 1.4 tonnes in $^{-1}=21.6 \mathrm{MPa}$ $\left(\sigma / \mu=4.9 \times 10^{-4}\right)$, at a temperature of $250^{\circ} \mathrm{C}=523 \mathrm{~K}$, $\left(T / T_{\mathrm{m}}=0.56\right)$. According to the summary of $\mathrm{Wu}$ and Sherby, this is the upper limiting stress for power-law creep. The data reported by Vogler and Blum [14] show a power law of about 4.5 at $533 \mathrm{~K}$ up to slightly above $20 \mathrm{MPa}$. For polycrystalline material, Hanson and Wheeler observed a rapid extension of about $3.7 \%$ in a period of about $20 \mathrm{~min}$. (This period seems to include the stage of transient creep which Andrade labeled $\beta$.) In this stage, slip bands were rare, but the grain boundaries became well marked.

This stage was followed by a period of steady creep lasting 3-40 h during which "no marked changes in microstruc- 
ture were observed". Then creep accelerated and substantial disruption of the crystal aggregate was observed.

In the third, steady-state, stage "slip bands are not formed except in unusual circumstances, but the crystal boundaries become well defined and broader." In this stage, after the initial rapid elongation of about $4 \%$, "the hardness remains constant, but the density slowly decreases." Though this change is slow, it seems to be much more rapid than that in the initial transient deformation.

These observations suggest strongly that, during this third stage of steady-state creep, deformation is concentrated near the grain boundaries.

The observations of Andrade and his group were made before it was generally accepted that the mechanisms of steady-state creep in the regions of power-law creep and power-law breakdown could well be different, and it is not easy to determine in which of these regimes the observations were made. Moreover, the results of different observations do not seem to be compatible. Andrade [12] found an exponential dependence of creep rate on stress for polycrystalline copper in the stress range $5-11 \mathrm{MPa}$ at $683 \mathrm{~K}$ while Myshlyaev et al. [11] found a clear power-law dependence of the creep rate for polycrystalline copper at $673 \mathrm{~K}$ in the same stress range. If in fact the regimes of power-law creep and power-law breakdown are physically distinct, it is not clear to which of these regions the observations of the Andrade group refer.

In the experiments of Andrade and Chalmers [21] and Gibbs and Ramlal [22] the metals studied were usually ones with a unique crystal axis, hexagonal cadmium or tetragonal tin. In both metals the electrical resistivity is greater along the unique axis than perpendicular to it, but in the case of cadmium the preferred glide directions are perpendicular to the axis, while the glide direction in tin is parallel to the axis. If tensile deformation of the polycrystalline aggregate occurs by crystallographic glide within the grains, the unique axis will rotate towards the tensile axis in the case of tin and away from it in the case of cadmium, leading to a decrease in the longitudinal resistivity in cadmium and an increase in tin, with no change in a cubic metal such as copper. In cadmium, the $\beta$ component led to a decrease in resistivity in roughly quantitative agreement with what would be expected as a result of crystallographic glide; the $\kappa$ component, steady-state creep, appeared to produce no decrease in resistivity and so to represent deformation with no systematic grain rotation. These results were largely confirmed by X-ray analysis [22]. Tin and copper showed the expected increase and constancy of longitudinal resistivity during creep.

This seems to be clear evidence that steady-state creep is occurring predominantly by a process which does not involve crystallographic glide and the consequent systematic rotation of the grains. Some sort of grain-boundary sliding seems to be indicated. This reinforces the implications of the observations of Hanson and Wheeler that steady-state creep does not produce slip lines or increase the hardness, but does reduce the density and cause the grain boundaries to become well defined and broaden.

Further evidence comes from the work of Andrade and Jolliffe [23] on "flow under simple shear". The test sample was in the form of a thick circular disc, clamped near the middle and round the edges. A deep circular groove was cut in one face, and the central and peripheral clamps were rotated with respect to one another. Plastic deformation, closely approximating to simple shear, occurred in the thin part of the disc remaining after the groove had been cut. The principal result was that, in lead at room temperature, the usual $\beta$ creep occurred, but steady-state creep occurred only after a period of accelerating creep associated with some form of recrystallization. Under these conditions of "simple shear", there was "no permanent creep even at temperatures at which, in tensile tests at constant stress, the creep is predominantly linear with time". They explained this by writing "the elimination of $\kappa$ flow with the present system of stressing by the fact that the surfaces across which the shearing forces are applied are not free, which suppresses the condition favourable for intergranular flow."

\section{Discussion}

The experimental evidence suggests that the evolution of creep under typical conditions occurs in four stages of which the third is steady-state, Andrade's $\kappa$, creep.

It is usually not clear whether observations were made in the regime of power-law creep or in the regime of power-law breakdown. While the data of $\mathrm{Wu}$ and Sherby on aluminium show a rather sharp transition between these two regimes, the data of Vogler and Blum on copper suggest a continuous transition between the two regimes. It is not clear whether the physical processes in the two regimes are the same or different. On the assumption that the physical processes in the two regimes are similar, the experiments show clear evidence that deformation is concentrated near the grain boundaries. According to Hanson and Wheeler "The first period of general stressing is followed by one in which extension is extremely slow: slip bands are not formed except in unusual circumstances, but the crystal boundaries become well defined and broader". There is little or no systematic lattice rotation, while the grains themselves undergo large homogeneous rotations (Fig. 36 of [24]). The Brinell hardness remains constant. Slip bands are scarce, but the grain boundaries become strongly marked. As Hanson [24] described the observations "The crystal boundaries themselves are smooth and clean-cut, when revealed by etching, and the surface markings in their neighbourhood produced by slow flow are considered to indicate complex distortions in the neighbourhood of the boundaries rather than the positions of the actual boundaries themselves. Hanson and Wheeler [20] described a crystal which was extended, re-polished, and extended a further $1.2 \%$ in $96 \mathrm{~h}$, when (their Figs. 5 and 6 ) it showed broad distortions at the grain boundaries. It was 
again re-polished "leaving a clear surface", which implies that there was no grain-boundary or triple-junction cavitation, and that the specimen was still in stage 3. A further extension of $2.6 \%$ in $48 \mathrm{~h}$ (representing an acceleration of creep) produced similar grain-boundary offsets. No visible slip lines were produced in either of these last strainings. To quote Andrade [12], "Broadly speaking, the $\beta$ flow is attributable to slip on glide planes within the crystal grains; it is a matter of adjustments within the grains. The permanent creep, on the other hand, is due to a relative adjustment of grains as a whole relative to one another, it is a boundary effect." Similarly Hanson [23] wrote “... complex stresses in the region of the grain junctions. They can relieve themselves from these increased stresses by localized plastic flow, also in a direction controlled by the applied load. A continual redistribution of the localized stresses at the boundary due to repetition of this process can account for the observed effects." The experimental evidence is thus that steady-state creep occurs by sliding at the grain boundaries. These boundaries remain almost atomically smooth, but continually migrate locally to relieve the local stresses.

Not only do the existing theories have difficulty in predicting rates of deformation as great as those observed, but they concentrate on processes occurring in the interior of the grains. The experimental evidence seems compelling that the deformation occurs predominantly at or close to the grain boundaries. A possible solution may be that the physical processes in power-law creep and in power-law breakdown are distinct: the former probably occurs by essentially homogeneous deformation within the grains, while the latter almost certainly occurs mainly by a process of grain-boundary sliding.

\section{Acknowledgements}

I am grateful to R.W. Cahn and J.-L. Martin for their suggestions.

\section{References}

[1] J.R. Spingarn, W.D. Nix, Acta Metall. 27 (1979) 171-177.

[2] J.L. Bocquet, G. Brebec, Y. Limoge, in: R.W. Cahn, P. Haasen (Eds.), Physical Metallurgy, vol. 1, North-Holland, Amsterdam, The Netherlands, 1996, pp. 535-668.

[3] T. Surholt, Chr. Herzig, Acta Mater. 45 (1997) 3817-3823.

[4] J. Weertman, J. Appl. Phys. 28 (1957) 362-364.

[5] J. Weertman, Trans. Am. Soc. Met. 61 (1968) 680-694.

[6] S.G.R. Brown, R.W. Evans, B. Wilshire, Mater. Sci. Technol. 3 (1987) 23-27.

[7] J. Weertman, in: B. Wilshire, D.R.J. Owen (Eds.), Creep and Fracture of Engineering Materials and Structures, Pineridge Press, Swansea, UK, 1984, pp. 1-13.

[8] B. Wilshire, C.J. Palmer, Scripta Mater. 46 (2002) 483-488.

[9] P. Feltham, J.D. Meakin, Acta Metall. 7 (1959) 614-627.

[10] H. Siethoff, K. Ahlborn, Scripta Metall. 20 (1986) 1445-1450.

[11] M.M. Myshlyaev, W.A. Stepanov, V.V. Shpeizman, Phys. Status Sol. (a) 8 (1971) 393-401.

[12] E.N. daC. Andrade, J. Iron Steel Inst. 171 (1952) 217-228.

[13] M.Y. Wu, O.D. Sherby, Acta Metall. 37 (1984) 1561-1572.

[14] S. Vogler, W. Blum, in: B. Wilshire, R.W. Evans (Eds.), Creep and Fracture of Engineering Materials and Structures, The Institute of Metals, London, UK, 1990, pp. 65-79.

[15] H.F. Moore, B.B. Betty, C.W. Dollins, Investigation of Creep and Fracture of Lead and Lead Alloys for Cable Sheathing, University of Illinois Bulletin, vol. 35, no. 102, The University of Illinois, Urbana, IL, USA, 1938.

[16] J.P. Poirier, Acta Metall. 26 (1978) 629-637.

[17] O.D. Sherby, J. Weertman, Acta Metall. 27 (1979) 387-400.

[18] A.D. Le Claire, in: S. Saimoto, G.R. Purdy, G.V. Kidson (Eds.), Solute-Defect Interaction-Theory and Experiment, Pergamon Press, Toronto, Canada, 1986, pp. 251-281.

[19] H. Luthy, A.K. Miller, O.D. Sherby, Acta Metall. 28 (1980) 169-178.

[20] D. Hanson, M.A. Wheeler, J. Inst. Met. 45 (1931) 229-264.

[21] E.N. daC. Andrade, B. Chalmers, Proc. R. Soc. A 138 (1932) 348374.

[22] R.E. Gibbs, N. Ramlal, Philos. Mag. 18 (1934) 949-956.

[23] E.N. daC. Andrade, K.H. Jolliffe, Proc. R. Soc. A 213 (1952) $3-26$.

[24] D. Hanson, Trans. Am. Inst. Min. Metall. Eng. 133 (1939) 1557. 